\title{
On the coupling of heavy mesons to pions in QCD
}

\author{
P. Colangelo and G. Nardulli \\ Dipartimento di Fisica, Univ.di Bari \\ I.N.F.N., Sezione di Bari
}

\begin{abstract}
A. Deandrea, N. Di Bartolomeo and R. Gatto
Département de Physique Théorique, Univ. de Genève

F. Feruglio

Dipartimento di Fisica, Univ. di Padova
\end{abstract}

UGVA-DPT 1994/06-856

BARI-TH/94-171

hep-ph/9406295

* Partially supported by the Swiss National Science Foundation 


\begin{abstract}
We compute the strong coupling constant $g_{P^{*} P \pi}$, with $P$ and $P^{*}$ respectively pseudoscalar and vector heavy mesons by using the QCD sum rules approach. Our computation is based on the evaluation of the time ordered product of currents between the vacuum and the soft pion state. The so-called parasitic terms are taken into account and give a contribution to the sum rule of the same order of the lowest lying state, while higher dimension non perturbative terms have small numerical effects. The infinite heavy quark mass limit is also examined.
\end{abstract}




\section{Introduction}

In this letter we shall address the problem of the determination of the strong coupling constant $g_{P^{*} P \pi}$, where $P$ and $P^{*}$ are respectively the lowest pseudoscalar and vector heavy meson made up of a light $q$ antiquark and a heavy quark $Q$. This coupling is defined by the strong amplitude

$$
<\pi^{-}(q) P^{o}\left(q_{2}\right) \mid P^{*-}\left(q_{1}, \epsilon\right)>=g_{P^{*} P \pi} \epsilon^{\mu} \cdot q_{\mu}
$$

and is of physical interest for a number of reasons. First, for $P=D$ and $P^{*}=D^{*}$, the decay amplitude (1.1) describes a process which is observed experimentally. Present data provide the upper bound:

$$
g_{D^{*} D \pi} \leq 13.8
$$

from $\Gamma_{\text {tot }}\left(D^{*+}\right)<131 \mathrm{KeV}$ [1] and $B R\left(D^{*+} \rightarrow D^{o} \pi^{+}\right)=30.8 \pm 0.4 \pm 0.8$ [2]. Second, it is commonly believed that the form factor $F_{1}\left(q^{2}\right)$ describing the semileptonic decay $B \rightarrow \pi \ell \nu$ is dominated, for $q^{2} \simeq q_{\max }^{2}=\left(m_{B}-m_{\pi}\right)^{2}$, by the $B^{*}$ pole; in this case $F_{1}\left(q_{\max }^{2}\right)$ would be given by the formula [3, 幽:

$$
F_{1}\left(q_{\max }^{2}\right) \simeq \frac{f_{B^{*}}}{4} \frac{g_{B^{*} B \pi}}{m_{\pi}+\left(m_{B^{*}}-m_{B}\right)}
$$

so that knowledge of the strong coupling $g_{B^{*} B \pi}$ would shed light on this decay process $\left(f_{B^{*}}\right.$ is the leptonic decay constant of $\left.B^{*}\right)$. Finally in the chiral effective field theories for heavy mesons that have recently received attention by a number of authors [5], [6], [7], [8], the above mentioned coupling is related to the interaction lagrangian 1

$$
\mathcal{L}_{i n t}=\frac{i}{2} g \operatorname{Tr}\left[H \gamma_{\mu} \gamma_{5}\left(\xi^{\dagger} \partial_{\mu} \xi-\xi \partial_{\mu} \xi^{\dagger}\right) \bar{H}\right]
$$

by the formula

$$
g_{P^{*} P \pi}=\frac{2 m_{P}}{f} g .
$$

The coupling constant $g$ appears in a number of calculations that make use of the chiral effective theories in the infinite heavy quark mass limit [8], [9], [10], [11].

The computation we shall describe in the next sections is based on the QCD sum rules approach 27. Some other QCD sum rules calculations of the strong coupling constant $g_{P^{*} P \pi}$ can be found in the literature [13], [14]. The authors of ref. [13] compute $g_{D^{*} D \pi}$ by considering two-point functions in an external pion field, whereas the authors of ref. 14 compute the coupling constant $g$ of eq.(1.4). We differ from these calculations in several aspects. First of all we compute $g_{D^{*} D \pi}, g_{B^{*} B \pi}$ and $g$, which allows us to discuss $1 / m_{Q}$ corrections to the asymptotic $m_{Q} \rightarrow \infty$ limit. Second we use the method of ref. 115 both in the evaluation of the correlators (the time ordered product of currents is taken between the vacuum and the pion state) and in the treatment of the so called parasitic terms, arising from non-diagonal transitions f they are present if, as in our case, one takes the

\footnotetext{
${ }^{1}$ We employ the notations of [5], where $\left.H=(1+\not)\right) / 2\left(P_{\mu}^{*} \gamma^{\mu}-P_{5} \gamma_{5}\right),\left(v\right.$ is the heavy meson velocity, $P_{\mu}^{*}$ and $P_{5}$ are annihilation operators of the heavy mesons $P^{*}$ and $P$ respectively) and $\xi^{2}=\Sigma=\exp i M / f$, with $M$ the $3 \times 3$ matrix of the Nambu-Goldstone pseudoscalar meson octet and $f=f_{\pi}$ in the chiral limit $\left(f_{\pi}=132 \mathrm{MeV}\right)$.

${ }^{2}$ For a review of QCD sum rules as applied to heavy masses see [12].

${ }^{3}$ See also [16]
} 
soft pion limit $q \rightarrow 0$, which implies $q_{1}=q_{2}$ and one has to take the single and not the double Borel transform. These differences alter the numerical result, as we shall discuss below; other differences with refs. [13], [14], arising from higher order non perturbative terms that we have included, have small numerical effects in the final results.

\section{QCD sum rule calculation of $g_{D^{*} D \pi}$ and $g_{B^{*} B \pi}$}

For definiteness we shall consider the off-shell process

$$
B^{*-}\left(\epsilon, q_{1}\right) \rightarrow \bar{B}^{0}\left(q_{2}\right)+\pi^{-}(q)
$$

the case of $D^{*} \rightarrow D \pi$ being completely analogous. We consider the correlator

$$
A_{\mu}(P, q)=i \int d x<\pi^{-}(q) \mid T\left(V_{\mu}(x) j_{5}(0) \mid 0>e^{-i q_{1} x}=A q_{\mu}+B P_{\mu}\right.
$$

where $V_{\mu}=\bar{u} \gamma_{\mu} b, j_{5}=i \bar{b} \gamma_{5} d, P=q_{1}+q_{2}$ and $A, B$ are scalar functions of $q_{1}^{2}, q_{2}^{2}, q^{2}$.

Both $A$ and $B$ satisfy dispersion relations and are computed, according to the QCD sum rules method, in two ways: either by saturating the dispersion relation by physical hadronic states or by means of the operator product expansion (OPE). We shall focus our attention on the invariant function $A$ that we compute in the soft pion limit $(q \rightarrow 0)$ and for large Euclidean momenta $\left(q_{1}^{2}=q_{2}^{2} \rightarrow-\infty\right)$.

Performing the OPE on $A$ we get the following result

$$
A=A^{(0)}+A^{(1)}+A^{(2)}+A^{(3)}+A^{(4)}+A^{(5)}
$$

with

$$
\begin{aligned}
& A^{(0)}=\frac{-1}{q_{1}^{2}-m_{b}^{2}}\left[m_{b} f_{\pi}+\frac{<\bar{u} u>}{f_{\pi}}\right] \\
& \left.A^{(1)}=-\frac{2}{3} \frac{1}{q_{1}^{2}-m_{b}^{2}} \frac{<\bar{u} u>\left[\frac{m_{b}^{2}}{f_{\pi}}-2\right]}{q_{1}^{2}-m_{b}^{2}}-2\right]-\frac{m_{o}^{2}<\bar{u} u>}{4 f_{\pi}\left(q_{1}^{2}-m_{b}^{2}\right)^{2}}\left[1-\frac{2 m_{b}^{2}}{q_{1}^{2} m_{b}^{2}}\right] \\
& A^{(2)}=\frac{m_{b} f_{\pi} m_{1}^{2}}{9\left(q_{1}^{2}-m_{b}^{2}\right)^{2}}\left[1+\frac{10 m_{b}^{2}}{q_{1}^{2}-m_{b}^{2}}\right] \\
& A^{(3)}=\frac{m_{0}^{2}<\bar{u} u>}{6 f_{\pi}}\left[\frac{1}{\left(m_{1}^{2}-m_{b}^{2}\right)^{2}}-\frac{2 m_{b}^{2}}{\left(q_{1}^{2}-m_{b}^{2}\right)^{3}}+\frac{\left.m_{b}^{2}\right)^{4}}{\left(q_{1}^{2}-m_{0}^{2}\right.}\right] \\
& A^{(4)}=\frac{1}{\left(q_{1}^{2}-m_{b}^{2}\right)^{2}}\left[\frac{2 f_{\pi}>}{4 f_{\pi}}+m_{b} f_{1}^{2}\right] \\
& A^{(5)}=\frac{m_{0}^{2}<\bar{u} u>}{6 f_{\pi}}\left[\frac{1}{\left(q_{1}^{2}-m_{b}^{2}\right)^{2}}-\frac{2 m_{b}^{2}}{\left(q_{1}^{2}-m_{b}^{2}\right)^{3}}\right] .
\end{aligned}
$$

In eqs.(2.4) $<\bar{u} u>$ is the quark condensate $\left(<\bar{u} u>=-(240 \mathrm{MeV})^{3}\right), m_{0}$ and $m_{1}$ are defined by the equations

$$
<\bar{u} g_{s} \sigma \cdot G u>=m_{0}^{2}<\bar{u} u>
$$

and

$$
<\pi(q)\left|\bar{u} D^{2} \gamma_{\mu} \gamma_{5} d\right| 0>=-i f_{\pi} m_{1}^{2} q_{\mu}
$$


and their numerical values are: $m_{0}^{2}=0.8 \mathrm{GeV}^{2}, m_{1}^{2}=0.2 \mathrm{GeV}^{2}$ [15, [17]. The origin of the different terms in (2.3) is as follows. $A^{(0)}$ is the leading term in the short distance expansion; $A^{(1)}, A^{(2)}$ and $A^{(3)}$ arise from the expansion of $V_{\mu}(x)$ at the first, second and third order in powers of $x ; A^{(4)}$ and $A^{(5)}$ arise from the expansion of the heavy quark propagator at the second order and from the zeroth and first term in the expansion of $V_{\mu}(x)$ respectively. We stress that we have considered all the operators with dimension $D \leq 5$ in the OPE of the currents appearing in (2.2); we also note that, with the obvious change $b \rightarrow c$, eq.(2.4) also applies to the amplitude for charm decay $D^{*} \rightarrow D \pi$.

Let us now evaluate the hadronic side of the sum rule, which can be obtained by writing for $A\left(0, q_{1}^{2}, q_{2}^{2}\right)$ the dispersion relation

$$
A\left(0, q_{1}^{2}, q_{2}^{2}\right)=\frac{1}{\pi^{2}} \int d s d s^{\prime} \frac{\rho\left(s, s^{\prime}\right)}{\left(s-q_{1}^{2}\right)\left(s^{\prime}-q_{2}^{2}\right)}+\text { subtractions } .
$$

We divide the integration region into three parts. The first region (I) is the square given by $m_{b}^{2} \leq s \leq s_{0}, m_{b}^{2} \leq s^{\prime} \leq s_{0}$; for $s_{0}$ small enough, (I) contains only the $B$ and $B^{*}$ poles, whose contribution is

$$
\begin{aligned}
A_{I}\left(0, q_{1}^{2}, q_{2}^{2}\right) & =\frac{f_{B} f_{B^{*}} m_{B}^{2}}{4 m_{b} m_{B^{*}}}\left[\frac{g_{B^{*} B \pi}\left(3 m_{B^{*}}^{2}+m_{B}^{2}\right)}{\left(q_{1}^{2}-m_{B^{*}}^{2}\right)\left(q_{2}^{2}-m_{B}^{2}\right)}+\right. \\
& \left.+\frac{g_{B^{*} B \pi}}{q_{1}^{2}-m_{B^{*}}^{2}}+\frac{3 f_{+}-f_{-}}{q_{2}^{2}-m_{B}^{2}}\right]
\end{aligned}
$$

where $f_{B}, f_{B^{*}}, f_{+}$and $f_{-}$are defined by

$$
\begin{gathered}
<0\left|A_{\mu}(0)\right| B(p)>=i p_{\mu} f_{B} \\
<0\left|V_{\mu}(0)\right| B^{*}(\epsilon, p)>=\epsilon_{\mu} f_{B^{*}} m_{B^{*}} \\
<\pi^{-}(q) \bar{B}^{0}\left(q_{2}\right) \mid B^{*-}\left(q_{1}\right)>=\left(f_{+} P_{\mu}-f_{-} q_{\mu}\right) \epsilon^{\mu} .
\end{gathered}
$$

Similar definitions hold for the $D^{*} \rightarrow D \pi$ decay.

The second (II) integration region in (2.7) is defined as follows: $m_{b}^{2} \leq s \leq s_{0}$ and $s^{\prime}>s_{0}$ or $m_{b}^{2} \leq s^{\prime} \leq s_{0}$ and $s>s_{0}$. In this region we can distinguish two contributions. The first one is obtained by coupling the vector current $V_{\mu}$ to the pion and the $B$ and the second one arises by coupling the pseudoscalar $j_{5}$ current to $\pi$ and $B^{*}$. The first term is as follows

$$
A_{I I}\left(0, q_{1}^{2}, q_{2}^{2}\right)=\frac{f_{B} m_{B}^{2}}{2 m_{b}} \frac{1}{q_{2}^{2}-m_{B}^{2}}\left[3 F_{1}-\frac{q_{2}^{2}}{q_{1}^{2}}\left(F_{0}-F_{1}\right)\right]
$$

where $F_{0}$ and $F_{1}$ are the usual form factors [18 describing the coupling of the vector current $V_{\mu}$ to the $B$ and $\pi$. We can safely assume that $F_{1}=F_{1}\left(q_{1}^{2}\right)$ is dominated by the $B^{*}$ pole [19], 20], 21], therefore the contribution from this form factor would be omitted to avoid double counting; assuming that the $q_{1}^{2}$ dependence of $F_{0}$ is given by the low lying $0^{+}$pole (whose mass we denote by $\bar{m}$ ) we obtain

$$
A_{I I}\left(0, q_{1}^{2}, q_{2}^{2}\right)=\frac{f_{B} m_{B}^{2} \bar{m}^{2} F_{0}(0)}{2 m_{b}} \frac{1}{q_{2}^{2}-m_{B}^{2}} \frac{1}{q_{1}^{2}-\bar{m}^{2}}
$$

As for the second term in the II region, it would be proportional to the $A_{0}\left(q_{2}^{2}\right)$ form factor (in the notations of ref.[18]). Assuming again the dominance of the low lying $0^{-} B$-pole 
we would obtain a term which coincides with $A_{I}$ and therefore it will be omitted to avoid double counting.

Let us finally consider the third (III) region in the $\left(s, s^{\prime}\right)$ plane, as defined by $s, s^{\prime}>s_{0}$. Assuming duality, in this region $A_{I I I}$ should coincide with the asymptotic $\left(q_{1}^{2}=q_{2}^{2} \rightarrow\right.$ $-\infty)$ limit of $(2.3)$; therefore the spectral function is given by:

$$
\rho_{I I I}\left(s, s^{\prime}\right)=\left[m_{b} f_{\pi}-\frac{<\bar{u} u>}{3 f_{\pi}}\right] \pi^{2} \theta\left(s-s_{0}\right) \theta\left(s^{\prime}-s_{0}\right) \delta\left(s-s^{\prime}\right) .
$$

Let us now discuss, before taking the single Borel transform of $A\left(0, q_{1}^{2}, q_{2}^{2}\right)$, the role of the terms (2.13), (2.14) and the single pole contributions to $A_{I}$ in (2.8) (in the literature they are called "parasitic" or non-diagonal terms). In general these terms contain new unknown quantities as well as several uncertainties; however we can exploit their different dependence on the Borel parameter $M^{2}$ as compared to the resonance term (the first contribution in (2.8) ); as a matter of fact, putting $q_{1}^{2}=q_{2}^{2}$ and taking the Borel transform, one gets the following sum rule:

$$
\begin{aligned}
\frac{\gamma}{M^{2}}+\lambda\left(M^{2}\right) & =\exp \left(\Omega / M^{2}\right)\left(\left[m_{b} f_{\pi}-\frac{<\bar{u} u>}{3 f_{\pi}}\right]\right. \\
& +\frac{1}{M^{2}}\left[-\frac{2<\bar{u} u>m_{b}^{2}}{3 f_{\pi}}+\frac{10 m_{b} f_{\pi} m_{1}^{2}}{9}+\frac{m_{0}^{2}<\bar{u} u>}{3 f_{\pi}}\right] \\
& +\frac{1}{2 M^{4}}\left[-\frac{10 m_{b}^{3} f_{\pi} m_{1}^{2}}{9}+\frac{m_{0}^{2}<\bar{u} u>m_{b}^{2}}{6 f_{\pi}}\right]+ \\
& \left.+\frac{m_{0}^{2}<\bar{u} u>m_{b}^{4}}{6 f_{\pi} M^{6}}\right)
\end{aligned}
$$

where

$$
\gamma=\frac{f_{B} f_{B^{*}} m_{B}^{2} g_{B^{*} B \pi}}{4 m_{b} m_{B^{*}}}\left(3 m_{B^{*}}^{2}+m_{B}^{2}\right)
$$

and $\Omega=\left(m_{B}^{2}+m_{B^{*}}^{2}-2 m_{b}^{2}\right) / 2$, while $\lambda\left(M^{2}\right)$ is given by

$$
\lambda\left(M^{2}\right)=d_{0}+d_{1} e^{-\delta / M^{2}}
$$

with

$$
\delta=s_{0}-m_{B}^{2}
$$

and $d_{0}, d_{1}$ unknown quantities. We note that $\lambda\left(M^{2}\right)$ takes into account all the possible parasitic terms, either deriving from eqs.2.13),(2.14) or from the last two terms on the r.h.s. of (2.8). We also note that in deriving (2.17) and (2.18) we put $\bar{m}^{2} \simeq s_{0}$, which, for the usually accepted values of $s_{0}\left(s_{0} \simeq 33-36 \mathrm{GeV}^{2}\right.$ for the $B, s_{0} \simeq 6-8 \mathrm{GeV}^{2}$ for the charm case) is a reasonable approximation. Moreover we put $m_{B}^{2} \simeq\left(m_{B}^{2}+m_{B^{*}}^{2}\right) / 2$ and $m_{D}^{2} \simeq\left(m_{D}^{2}+m_{D^{*}}^{2}\right) / 2$

We can now differentiate (2.15) in the variable $1 / M^{2}$ so that we remain with a new sum rule (NSR) for the variable $\gamma+\partial \lambda / \partial\left(1 / M^{2}\right)$. Since $\gamma$ does not depend on $M^{2}$, we differentiate again this equation, so that we obtain an equation in the unknown quantity $d_{1}$. Substituting this expression in NSR we finally obtain the following sum rule for $g_{B^{*} B \pi}$ :

$$
\gamma=\exp \left[\frac{\Omega}{M^{2}}\right]\left\{\Omega ( 1 + \frac { \Omega } { \delta } ) \left[m_{b} f_{\pi}-\frac{<\bar{u} u>}{3 f_{\pi}}\left(1+\frac{2 m_{b}^{2}}{M^{2}}\right)+\right.\right.
$$




$$
\begin{aligned}
& \left.+\frac{10 f_{\pi} m_{1}^{2} m_{b}}{9 M^{2}}\left(1-\frac{m_{b}^{2}}{2 M^{2}}\right)+\frac{m_{0}^{2}<\bar{u} u>}{3 f_{\pi} M^{2}}\left(1+\frac{m_{b}^{2}}{4 M^{2}}+\frac{m_{b}^{4}}{2 M^{4}}\right)\right]+ \\
& +\frac{2 \Omega+\delta}{\delta}\left[-\frac{2<\bar{u} u>m_{b}^{2}}{3 f_{\pi}}+\frac{10 f_{\pi} m_{1}^{2} m_{b}}{9}\left(1-\frac{m_{b}^{2}}{M^{2}}\right)+\right. \\
& \left.+\frac{m_{0}^{2}<\bar{u} u>}{6 f_{\pi}}\left(2+\frac{m_{b}^{2}}{M^{2}}+\frac{3 m_{b}^{4}}{M^{4}}\right)\right]-\frac{10 f_{\pi} m_{1}^{2} m_{b}^{3}}{9 \delta}+ \\
& \left.+\frac{m_{0}^{2}<\bar{u} u>m_{b}^{2}}{6 f_{\pi} \delta}\left(1+\frac{6 m_{b}^{2}}{M^{2}}\right)\right\}
\end{aligned}
$$

The analysis of the sum rule follows the usual criteria adopted in the literature: we check the existence of a region in $M^{2}$ where the OPE displays a convergent structure and where the highest states give a small contribution, as we discuss in the following section. Using $m_{b}=4.6 \mathrm{GeV}$ and $m_{c}=1.34 \mathrm{GeV}$ [12 for the heavy quark masses we obtain, in the region $M^{2} \geq 30 \mathrm{GeV}^{2}$ for $B$ and $M^{2} \geq 8 \mathrm{GeV}^{2}$ for $D$ :

$$
\begin{aligned}
f_{B} f_{B^{*}} g_{B^{*} B \pi} & =0.56 \pm 0.10 \mathrm{GeV}^{2} \\
f_{D} f_{D^{*}} g_{D^{*} D \pi} & =0.34 \pm 0.04 \mathrm{GeV}^{2}
\end{aligned}
$$

the uncertainty is mainly due to the variation of the threshold $s_{0}$. It should be noticed that the parasitic terms represent a relevant fraction $(40-50 \%)$ of the sum rule.

The strong couplings $g_{P^{*} P \pi}$ can be derived from (2.20, 2.21) once the leptonic constants are known. Some authors [13, 14] suggest that in eqs.(2.15) one should use as an input the leptonic constants obtained by two-point QCD sum rules at order $\alpha_{s}=0$, to be consistent with the calculation of the correlator (2.2) carried out at the same order in $\alpha_{s}$. Using $f_{D}=170 \pm 10 \mathrm{MeV}, f_{D^{*}}=220 \pm 24 \mathrm{MeV}, f_{B}=150 \pm 20 \mathrm{MeV}, f_{B^{*}}=190 \pm 10 \mathrm{MeV}$, we obtain:

$$
\begin{aligned}
& g_{B^{*} B \pi}=20 \pm 4 \\
& g_{D^{*} D \pi}=9 \pm 1
\end{aligned}
$$

It should be noticed, however, that in a sum rule the $O\left(\alpha_{s}\right)$ terms appear in the coefficients of the condensates, where they usually give small corrections, and in the perturbative contribution, where they are sizeable in the case of the leptonic constants. On the other hand, in the determination of $g_{P^{*} P \pi}$ the perturbative contribution (given by the operator 1 of the expansion of the correlator in eq.(2.2) calculated between the vacuum and the pion state) vanishes at all orders in $\alpha_{s}$; this is evident if one considers two-point functions in the external pion field [13], [14]. For this reason it seems to us that $\alpha_{s}$ corrections could be included in the calculation of the leptonic constants, where they are relevant, whereas they could be safely neglected in all other quantities. Using the results obtained by two-point function QCD sum rules (with $O\left(\alpha_{s}\right)$ corrections) $f_{B}=180 \pm 30 \mathrm{MeV}$, $f_{D}=195 \pm 20 \mathrm{MeV}$ 团 and $f_{B^{*}}=213 \pm 34 \mathrm{MeV}, f_{D^{*}}=258 \pm 26 \mathrm{MeV}$ obtained by a direct calculation in [23] and by an analysis of the heavy quark spin symmetry breaking in [24] we get for $g_{P^{*} P \pi}$ :

$$
\begin{aligned}
& g_{B^{*} B \pi}=15 \pm 4 \\
& g_{D^{*} D \pi}=7 \pm 1
\end{aligned}
$$

\footnotetext{
${ }^{4}$ For a review see 12,22 .
} 
Using the value in eq.(2.25) we predict: $\Gamma\left(D^{*+} \rightarrow D^{o} \pi^{+}\right)=10 \pm 3 \mathrm{KeV}$. Moreover, assuming the dominance of the $B^{*}$ pole in the form factor $F_{1}\left(q^{2}\right)$ for $B \rightarrow \pi \ell \nu$, we obtain, using (2.24): $F_{1}(0)=0.30 \pm 0.07$ in agreement with other QCD sum rules calculations of this form factor [19, 20].

Let us discuss an interesting consequence of our result. In a recent paper 25] it has been suggested that the scaling laws of the heavy quark effective theory can be used to derive the ratio $\left|V_{u b} / V_{c d}\right|$ from the comparison of the spectra $B \rightarrow \pi \ell \nu$ and $D \rightarrow \pi \ell \nu$ near the zero recoil point. A crucial ingredient in this strategy is the ratio

$$
R_{B D}=\sqrt{\frac{m_{D}}{m_{B}}} \frac{f_{B^{*}}}{f_{D^{*}}} \frac{g_{B^{*} B \pi}}{g_{D^{*} D \pi}}
$$

which is equal to 1 in the infinite heavy quark mass limit. Our results point to a value:

$$
R_{B D}=1.06-1.11
$$

depending on the role of $\alpha_{s}$ corrections (the highest value corresponds to $\alpha_{s}=0$ ); this $O(10 \%)$ correction should be taken into account when determining $V_{u b}$ in this approach.

\section{The limit $m_{Q} \rightarrow \infty$}

The infinite heavy quark mass limit $\left(m_{B} \rightarrow \infty\right)$ in eq.(2.15) can be performed according to the procedure already applied to leptonic constants [26], [27] and form factors [12]. In terms of low energy parameters the quantities in eq.(2.15) can be written as follows:

$$
\begin{aligned}
m_{B} & =m_{b}+\omega \\
m_{B^{*}}-m_{B} & =\mathcal{O}\left(\frac{1}{m_{b}}\right) \\
f_{B} & =f_{B^{*}}=\frac{\hat{F}}{\sqrt{m_{b}}}
\end{aligned}
$$

$\omega$ represents the binding energy of the meson, which is finite in the limit $m_{b} \rightarrow \infty$; the scaled leptonic constant $\hat{F}$ is independent of $m_{b}$ (if one neglects a small logarithmic dependence). $\hat{F}$ has been computed by QCD sum rules: for $\omega=0.625 \mathrm{GeV}$ and the threshold $y_{0}=\frac{s_{0}-m_{b}^{2}}{2 m_{b}}$ in the range $1.1-1.4 \mathrm{GeV}$ the result is $\hat{F}=0.30 \pm 0.05 \mathrm{GeV}^{3 / 2}$ (at the order $\alpha_{s}=0$ ) [24 and $\hat{F}=0.41 \pm 0.04 \mathrm{GeV}^{3 / 2}$ (including radiative corrections) [27].

The sum rule for $g$ in eq.(1.5) can be derived from (2.15) after having expressed the Borel parameter $M^{2}$ in terms of the low energy parameter $E$ : $M^{2}=2 m_{b} E$. One readily obtains:

$$
\begin{aligned}
g & =\frac{f_{\pi}^{2}}{\hat{F}^{2}} e^{\omega / E}\left\{\frac { 1 } { y _ { 0 } - \omega } \left[\omega^{2}\left(1-\frac{<\bar{u} u>}{3 f_{\pi}^{2} E}-\frac{5 m_{1}^{2}}{36 E^{2}}-\frac{m_{0}^{2}<\bar{u} u>}{72 E^{3} f_{\pi}^{2}}\right)+\right.\right. \\
& \left.-2 \omega\left(\frac{<\bar{u} u>}{3 f_{\pi}^{2}}+\frac{5 m_{1}^{2}}{18 E}+\frac{m_{0}^{2}<\bar{u} u>}{24 E^{2} f_{\pi}^{2}}\right)-\frac{5 m_{1}^{2}}{18}-\frac{m_{0}^{2}<\bar{u} u>}{12 E f_{\pi}^{2}}\right]+ \\
& +\omega\left(1-\frac{<\bar{u} u>}{3 f_{\pi}^{2} E}-\frac{5 m_{1}^{2}}{36 E^{2}}-\frac{m_{0}^{2}<\bar{u} u>}{72 E^{3} f_{\pi}^{2}}\right)-\frac{<\bar{u} u>}{3 f_{\pi}^{2}}+ \\
& \left.-\frac{5 m_{1}^{2}}{18 E}-\frac{m_{0}^{2}<\bar{u} u>}{24 E^{2} f_{\pi}^{2}}\right\}
\end{aligned}
$$


This sum rule must be studied in the region of the external parameter $E$ where the OPE is assumed to converge and where the contribution of higher resonances is small ("duality" region). By imposing the constraint that the various terms of the OPE in (3.2) display a hierarchical structure, according to the dimension, we get that $E$ should be larger than $1-2 \mathrm{GeV}$. As for the contribution of higher resonances, it is controlled in (3.2) by the parasitic term. It can be shown that this contribution, for values of the parameters in the range chosen above, is numerically of the same size of the contribution of the lowest lying states for any value of $E$. A prediction for $g$ can be obtained by studying the stability plateau for (3.2), which corresponds to $E \geq 4 \mathrm{GeV}$.

We obtain:

$$
\hat{F}^{2} g=0.035 \pm 0.008 G e V^{3} .
$$

Therefore at the order $\alpha_{s}=0$ our result is:

$$
g=0.39 \pm 0.16
$$

As we have discussed already, we could introduce a partial set of $O\left(\alpha_{s}\right)$ corrections, i.e. those induced by $\hat{F}$, which should represent the largest part of such corrections; in this case we get:

$$
g=0.21 \pm 0.06
$$

The difference between (3.4) and (3.5) reflects the well known important role of radiative corrections in the determination of $f_{B}$ by QCD sum rules in the $m_{Q} \rightarrow \infty$ limit 28]. However it should be noticed that the value of $g$ extracted from (1.5) using (2.22) or (2.24) is in the range $0.19-0.25$, which, if $1 / m_{Q}$ corrections are not anomalously large, points to the smaller value in (3.5).

\section{Conclusions}

The strong couplings $g_{P^{*} P \pi}$ play an important role in heavy meson phenomenology. They are directly related to the decays of $P^{*}$ into $P+\pi$ and they are expected to be important for the semileptonic decays of $P$ into a pion. They are also important inputs in the effective chiral lagrangians for heavy mesons. We have estimated the coupling constants $g_{D^{*} D \pi}$ and $g_{B^{*} B \pi}$ in the soft pion limit and for finite heavy quark mass. Our calculation uses QCD sum rules as applied to the correlator of a vector and a pseudoscalar current between pion and vacuum. It is done for $b$ and $c$ states, thus allowing to examine the validity of the infinite heavy quark mass limit. We have considered the contributions of all the operators up to dimension five to the operator product expansion: it turns out that the higher dimension ones give small contributions. The soft pion limit and the single Borel transformation give rise to parasitic terms which are not exponentially suppressed. They give an essential correction to the value of the coupling, of the order of the lowest resonance contribution. The duality region, where the OPE shows convergence and the higher resonances give small contributions, is found for values of $M^{2}$, the Borel parameter, higher than those usually found in the ordinary sum rules for semileptonic decays of heavy

mesons. This could indicate a slow convergence of the OPE; nevertheless we have checked that in this region the parasitic terms do not give a contribution larger than $50 \%$ to the sum rule.

As we discussed before, the absence of the pure perturbative term in the OPE, where usually radiative corrections are relevant, seems to indicate that the largest $\alpha_{s}$ corrections 
in the sum rule could be taken into account by using the radiative corrected values of the leptonic decay constants. In the case of finite heavy quark mass, the difference between the two choices (i.e. leptonic decay constants with or without $\alpha_{s}$ corrections) is within the uncertainties of our approach, while in the limit of infinite heavy quark mass there is a factor of two. Our numerical calculation of the sum rule, in the case when strong radiative corrections in the values of the leptonic constants are included, leads to values $g_{B^{*} B \pi}=15 \pm 4$ and $g_{D^{*} D \pi}=7 \pm 1$. Without including these radiative corrections we are lead to $g_{B^{*} B \pi}=20 \pm 4$ and $g_{D^{*} D \pi}=9 \pm 1$. The former case leads for the weak vector

form factor between $B$ and $\pi, F_{1}(0)$, to a value of $0.30 \pm 0.07$, in agreement with previous calculations.

\section{Acknowledgements}

We would like to thank R. Casalbuoni for discussions during the early stage of this work. 


\section{References}

[1] ACCMOR Collab., S.Barlag et al., Phys. Lett. B 278 (1992) 480.

[2] CLEO Collab., F.Butler et al., Phys Rev. Lett. 69 (1992) 2041.

[3] S.Nussinov and W.Wetzel, Phys. Rev. D 36 (1987) 139.

[4] L.Wolfenstein, Phys. Lett. B 291 (1992) 177.

[5] M.B.Wise, Phys. Rev. D 45 (1992) R2188.

[6] T.-M.Yan, H.-Y.Cheng, C.-Y.Cheung, G.-L.Lin, Y.C.Lin and H.-L.Yu, Phys. Rev. D 46 (1992) 1148.

[7] G.Burdman and J.F.Donoghue, Phys. Lett. B 280 (1992) 287.

[8] R.Casalbuoni, A.Deandrea, N.Di Bartolomeo, F.Feruglio, R.Gatto and G.Nardulli, Phys. Lett. B 299 (1993) 139.

[9] P.Cho and H.Georgi, Phys. Lett. B 296 (1992) 408; B 300 (1993) 410 (E).

[10] J.F.Amundson, C.G.Boyd, E.Jenkins, M.Luke, A.V.Manohar, J.L.Rosner, M.J.Savage and M.B.Wise, Phys. Lett. B 296 (1992) 415.

[11] P.Colangelo, F.De Fazio and G.Nardulli, Phys. Lett. B 316 (1993) 555.

[12] P.Colangelo, G.Nardulli and N.Paver, Proceedings of the ECFA Workshop on a European B-Meson Factory, R.Aleksan and A.Ali Eds., ECFA 93/151, pag.155.

[13] V.L.Eletski and Y.I.Kogan, Z.Phys. C 28 (1985) 155.

[14] A.G.Grozin and O.I.Yakovlev, preprint BUDKERINP-94-3 (hep-ph/9401267)

[15] V.A.Novikov, M.A.Shifman, A.I.Vainshtein, M.B.Voloshin and V.I.Zakharov, Nucl. Phys. B 237 (1984) 525.

[16] B.L.Ioffe and A.V.Smilga, Nucl. Phys. B232 (1984) 109.

[17] A.R.Zhitnitskii, I.R.Zhitnitskii and V.L.Chernyak, Sov. J. Nucl. Phys. 38 (1983) 645.

[18] M.Wirbel, B.Stech and M.Bauer, Z.Phys. C 29 (1985) 637.

[19] P.Ball, Phys. Rev. D 48 (1993) 3190.

[20] P.Colangelo and P.Santorelli, preprint BARI-TH-93-163 (hep-ph/9312258)

[21] B.Grinstein and P.Mende, preprint BROWN-HET-928; B.Grinstein and P.Mende, preprint BROWN-HET-930, SSCL-PREPRINT-549.

[22] C.A.Dominguez, preprint SISSA Ref 139/93/EP (hep-ph/9309260), to appear in the Proceedings of the Third Workshop on the Tau-Charm Factory, J.Kirkby and R.Kirkby Eds. Editions Frontieres, 1994.

[23] P.Colangelo, G.Nardulli, A.A.Ovchinnikov and N.Paver, Phys. Lett. B 269 (1991) 204. 
[24] M.Neubert, Phys. Rev. D 46 (1992) 1076.

[25] G.Burdman, Z.Ligeti, M.Neubert and Y.Nir, Phys. Rev. D 49 (1994) 2331.

[26] E.Shuryak, Nucl. Phys. B 198 (1982) 83.

[27] M.Neubert, Phys. Rev. D 45 (1992) 2451.

[28] D.J.Broadhurst and A.G.Grozin, Phys. Lett. B 274 (1992) 421. 